\title{
Mortality Risk Assessment of ICU Cardiovascular Patients Using Physiological Variables
}

\author{
Mohammad Karimi Moridani ${ }^{1, *}$, Seyed Kamaledin Setarehdan ${ }^{2}$, Ali Motie Nasrabadi ${ }^{3}$, \\ Esmaeil Hajinasrollah ${ }^{4}$
}

\author{
${ }^{1}$ Department of Biomedical Engineering, Science and Research Branch, Islamic Azad University, Tehran, Iran \\ ${ }^{2}$ Control and Intelligent Processing Centre of Excellence, School Electrical and Computer Engineering, College of Engineering, \\ University of Tehran, Tehran, Iran \\ ${ }^{3}$ Biomedical Engineering Department, Faculty of Engineering, Shahed University, Tehran, Iran \\ ${ }^{4}$ Loghman Medical Center, Shahid Beheshti University of Medical Sciences, Tehran, Iran \\ *Corresponding Author: mkarimi.bme@gmail.com
}

Copyright (C) 2013 Horizon Research Publishing All rights reserved.

\begin{abstract}
Recognition of mortality-related factors in intensive care units (ICUs) could increase the efficiency and effectiveness of these units. The purpose of this research is to investigate the recorded data of the patients admitted in ICU with clinical analysis, in order to find indices of mortality. The long-term goal of this study is to develop an algorithm that is able to anticipate the mortality risk of ICU patients. Extracted features included time and frequency domain analysis of ECG and some of the physiological variables. The results showed that heart rate variability (HRV) and blood pressure are the most important parameters in ICU mortality risk assessment and anticipation for cardiovascular patients.
\end{abstract}

Keywords Intensive Care Unit, Mortality Risk, Heart Rate Variability

\section{Introduction}

Heart rate variability (HRV), a marker of autonomic tone, has been extensively studied after myocardial infarction and has been established as a potent predictor of mortality [1].

The primary role of the intensive care units is to monitor and stabilize the vital functions of the patients with life-threatening conditions. In order to aid ICU nurses and intensities with this work, scoring systems have been developed to express the overall state of an ICU patient as a numerical value, that is then used to develop a classification rule, which classifies a patient as being at risk or not. Such scoring systems typically depend on the parameters that are estimated from a database of cases, but one of their features is that, often, they have missing values [2]. One suggestion as to why a patient attributes remains unrecorded is that an intensity assumes the variable to be clinically normal on the basis of other observations, therefore, not worthy of confirmation. Although this clinical-normality assumption has been criticized [3], the mortality rate is higher in those patients with completed records. Since abnormal physiological values are associated with increased risk, it has been argued that it supports the clinical-normality assumption. In addition, we suspect that there are some random omissions due to the pressure of work within an ICU; thus, it may be the case that the incompleteness of an ICU data set is due to a mixture of different missing-data mechanisms.

Identification of priorities in intensive care units is a problem of the high complexity due to dysfunction in patients' vital organs, such as blood, lung and kidney, which often undergo rapid changes in the mentioned wards. Therefore, nurses should be able to make decisions with respect to priorities to resolve this problem.

Likewise in a study frequency domain parameters were found to be decreased after MI but the Holter recordings were done for 20 minutes only and time domain parameters were not described [4]. Some of the data reported decrease in time and frequency domain indices after MI but the Holter recordings were done 2-4 weeks after the onset of symptoms [5-6].

Other studies documented decreased in HRV early after MI, but either 24 hours Holter recording was not done or all of the time and frequency domain parameters were not analyzed [8-9]. Correlation amongst various HRV indices has also been documented in research literature [5-7].

Therefore, we planned a study to compare the time and frequency domain parameters of surviving and dead of our population that monitored within 48 hours of the admission. Our study also focused on the correlation between some of these parameters in survival individuals and dead.

This paper is organized as follows: Section 2 describes data sets that were used, preprocessing, feature extraction and introduction of the proposed algorithm for mortality risk assessment.

Section 3 presents obtained result based on proposed algorithm, and Section 4 summarizes the conclusions. 


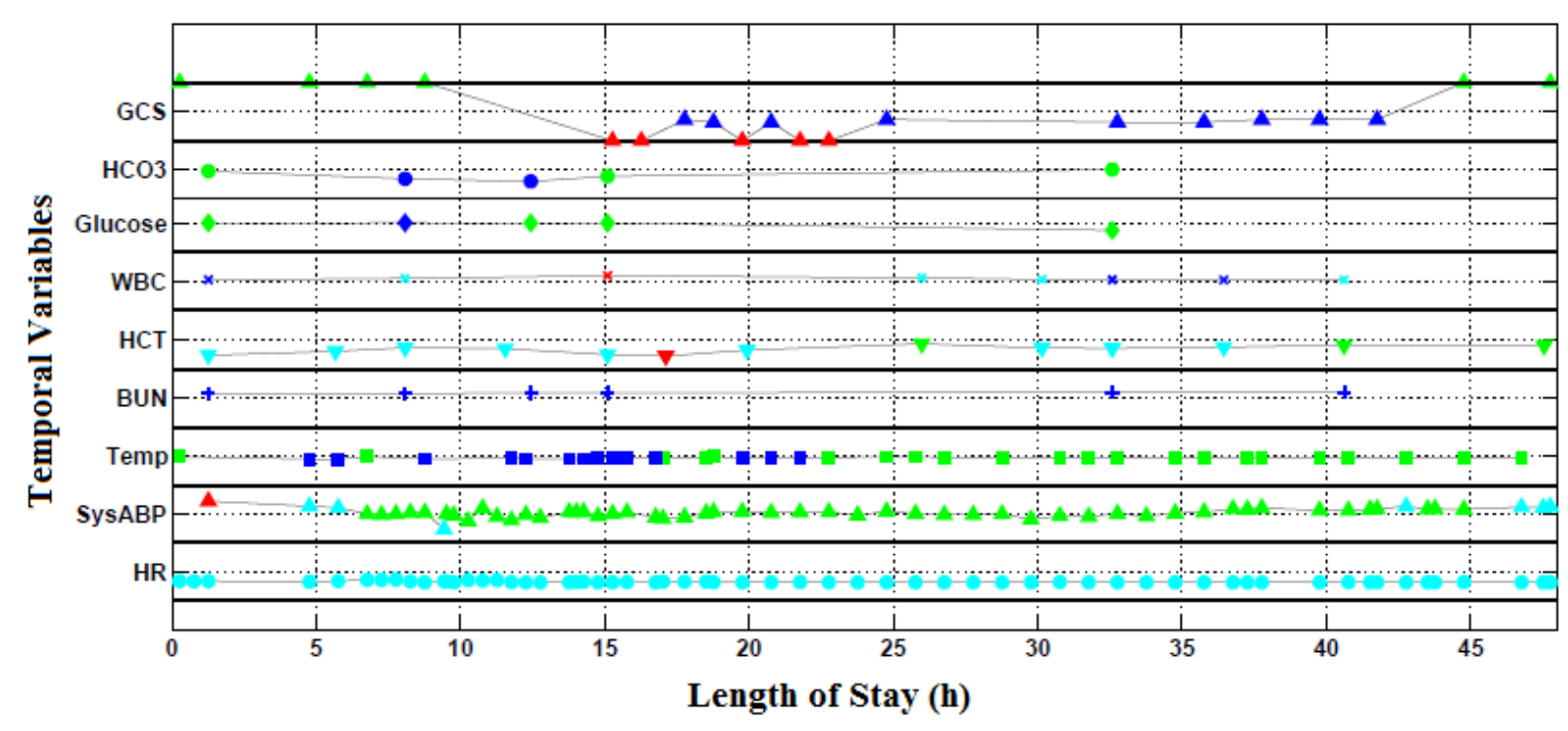

Figure 1. Length of stay for patients in the first 48 hours

\section{Materials and Methods}

\subsection{Dataset}

All datasets comprised of 100 cardiovascular patients include 55 males and 45 females with a mean (standard deviation) age of 46 (6) years, uncorrected height, and uncorrected initial weights 169.5 (17.1) centimeters, and $81.2(23.8) \mathrm{kg}$ respectively were analyzed in this study. The largest number of patients was admitted to the medical ICU $(35.8 \%)$, followed by the surgical $(28.4 \%)$, cardiac surgery recovery $(21.1 \%)$, and coronary $(21.1 \%)$ ICUs. We select 100 cardiovascular patients include bradycardia, tachycardia, fibrillation and ischemic [10] of different ICU types explained above.

The data recorded were include age, height, weight, HR variables (RR intervals, mean HR, maximum beat per minute (MBPM), LF/HF), systolic arterial blood pressure (SysABP), Temperature (Temp), respiratory rate (RespRate), mechanical ventilation (MechVent), Urine, blood urea nitrogen (BUN), hematocrit (HCT), white blood cell (WBC), glucose, $\mathrm{K}, \mathrm{Na}, \mathrm{HCO}_{3}$, Glasgow coma scale (GCS), and other variables that were added diastolic arterial blood pressure (DiasABP), Cholesterol, Creatinine, and $\mathrm{SaO}_{2}$ were recorded. Figure 1 shows an example of length of stay for patients in the first 48 hours of an ICU.

\subsection{Data Preprocessing}

Prior to using the dataset, each subjects' measurements were assessed using domain knowledge and distributional assumptions. That is, we impute physiologically implausible values of certain variables, either assigning the entry is unknown or substituting it with a physiologically valid entry. An example substitution would be converting height from an implausible value (e.g. 65, presumably an erroneous recording using inches) to a plausible value (e.g.
165 centimeters). This variable specific pre-processing was performed for age, height, diastolic arterial blood pressure, systolic arterial blood pressure, heart rate, Nitrogen, partial pressure of oxygen, temperature, white blood cell count and weight. Missing data were present in the data and handled by the model [11].

\subsection{Feature Extraction}

We converted each patient's time-stamped temporal variables into scalar features. For the static variables; age, initial weight, height, and gender, this was done by directly treating the value present as a feature. For the temporal variables, this was done by extracting the minimum, maximum, mean, first, last, and number of values for each time stamped variable. Thus, for each temporal variable, six features were extracted.

\subsection{Proposed Algorithm}

The paired sample test was used for analysis of continuous variables and Chi Square (C2) test was used for analysis of categorical variables. The $p$ value of less than 0.05 was considered as statistically significant.

The paired sample test is probably the most widely used parametric tests. A single sample t-test is used to determine whether the mean of a sample is different from a known average. A 2-sample t-test is used to establish whether a difference occurs between the means of 2 similar data sets. The t-test uses the mean, standard deviation, and number of samples to calculate the test statistic [12].

The chi-squared test is usually used to compare multiple groups where the input variable and the output variable are binary. The Chi-Square Formula is as Equation (1).

$$
X^{2}=\sum_{i=1}^{r} \sum_{j=1}^{c} \frac{\left(o_{i j}-E_{i j}\right)^{2}}{E_{i j}}
$$

In Equation (1), $r$ stands for "row", $c$ stands for 
"column", $O$ stands for "observed value", and $E$ stands for "expected value".

Our goal is to identify individual features in such datasets that distinguish between the two groups, i.e. features whose abundance in the two groups is different. Furthermore, we develop a statistical measure of confidence in the observed differences.

The input to our method can be represented as a Feature Abundance Matrix whose rows correspond to specific features, and whose columns correspond to individual samples. The cell in the $i^{\text {th }}$ row and $j^{\text {th }}$ column is the total number of observations of features $i$ in sample $j$ (Figure 2). Every distinct observation is represented only once in the matrix, i.e. overlapping features are not allowed (the rows correspond to a partition of the set of sequences). In Figure 2, each row represents a specific feature, while each column represents a subject. The $i^{\text {th }}$ feature in the $j^{\text {th }} \operatorname{subject~}(\mathrm{c}(\mathrm{i}, \mathrm{j}))$ is recorded in the corresponding cell of the matrix. If there are $g$ subjects in the first group, they are represented by the first $g$ columns of the matrix, while the remaining columns represent subjects from the second group [13].

$$
\begin{aligned}
& \begin{array}{llll}
\mathrm{S} 1 & \mathrm{~S} 2 & \ldots & \mathrm{S}(\mathrm{N}-1)
\end{array}
\end{aligned}
$$

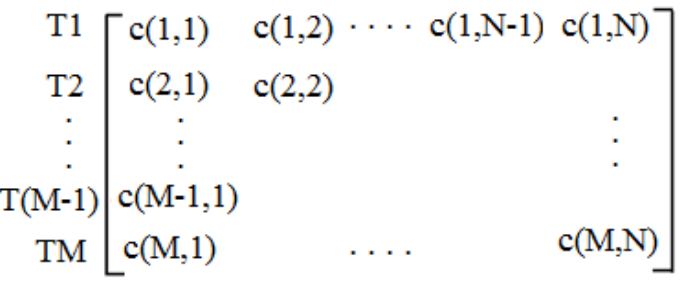

Figure 2. Format of the feature abundance matrix

Figure 3 shows a block diagram for mortality risk assessment. In the first step, we collect a database of patients that were admitted in ICU. Then, the preprocessing analysis includes removing the patients under 18 years, data filtering; normalization based on each patient was applied. Preprocessing is an important stage in a proposed algorithm because it is directly related to test results. The next step of this algorithm is feature extraction. The proposed algorithm based on the extracted features computes mortality risk in admitting patients in ICU.

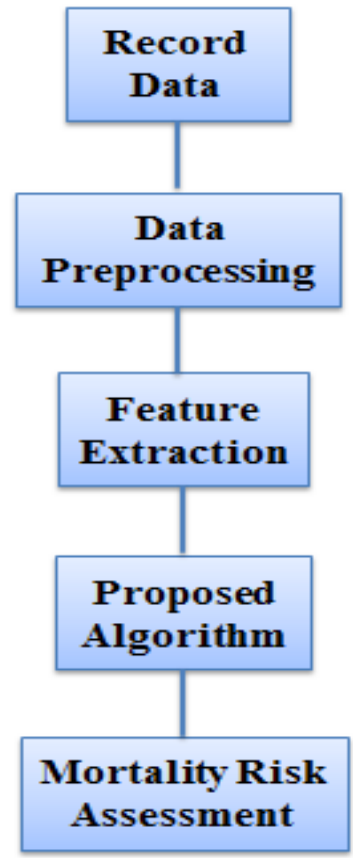

Figure 3. Block diagram for mortality risk assessment

\section{Results}

The experimental results are presented in Table 1. The clinical parameters were reported as mean \pm standard deviation or percent.

According to the results, it can be seen that of mentioned features, RR interval, $\mathrm{LF} / \mathrm{HF}$ and mean systolic blood pressure of 5 hours before death (43 hours after admission in ICU) were significantly higher than the same times in group $1,(p<0.05)$. The significant changes for each feature are marked in bold. However, as it can be seen in the Table 1, fluctuations in heart rate in 5 hours before death (group 1) are less than the first 43 hours (group 2). Therefore, heart rate variability is reduced in group 2 ..

Table 1. Comparison of physiological variables between 0-43 hours (group 1) and 43-48 hours (group 2) after admission.

\begin{tabular}{|c|c|c|c|}
\hline Feature & group1 & group2 & $\mathrm{P}$ value \\
\hline RR (ms) & $850.81 \pm 110$ & $750.87 \pm 30$ & $\mathrm{P}=0.012$ \\
\hline Mean HR & $70.49 \pm 12.1$ & $80.16 \pm 4$ & $\mathrm{P}=0.015$ \\
\hline MBPM & $80 \pm 5.31$ & $110.43 \pm 5.3$ & $\mathrm{P}=0.021$ \\
\hline LF/HF & $0.72 \pm 0.36$ & $1.78 \pm 0.7$ & $\mathrm{P}=0.042$ \\
\hline Mean SysABP & $112 \pm 7.72$ & $162 \pm 1.45$ & $\mathrm{P}=0.013$ \\
\hline Mean DiasABP & $71 \pm 6.69$ & $99 \pm 4.99$ & $\mathrm{P}=0.033$ \\
\hline Mean GCS & $14.2 \pm 0.8$ & $8 \pm 3.86$ & $\mathrm{P}=0.026$ \\
\hline Mean PH & $7.45 \pm 0.12$ & $7.3 \pm 1.62$ & $\mathrm{P}=0.09$ \\
\hline Mean RespRate & $17.36 \pm 2.75$ & $34.53 \pm 5.23$ & $\mathrm{P}=0.02$ \\
\hline
\end{tabular}


As the greater percentage of patients in this study consisted of patients with tachycardia disease (45\%), mean of heart rate is high, but what is more important is the standard deviation on of the heart rate in group 2 .

As the systolic and diastolic blood pressure in group 2 is much more than group 1, it can be concluded that this feature is an important factor in mortality prediction.

\section{Discussion}

Analysis of physiological variables shows that there are significant differences in mean HR, MBPM and mean systolic blood pressure mean RespRate of 5 hours before death. It means that this period of time and indices can lead us to find the golden time to recover the life of patients with reversible medical conditions.

We can expect that these measures and period of time enable early detection, anticipation or maybe management to recover the ICU patients. Prospective studies include analysis of other factors such as effect of gender and family history of the important diseases that might have effects on mortality are necessary to confirm the present results and establish guidelines for developing a robust prediction system.

In the present study, our main focus was based on the effect of the predictive value of heart rate variability and blood pressure for mortality from coronary heart disease, and from all causes was investigated in the general population. Moreover, analysis of patients' behavior during this period of time can lead us to find chaotic behaviors of HRV, blood pressure and other features that are robust indices of mortality prediction.

\section{REFERENCES}

[1] Psychari SN, Apostolou TS, Iliodromitis EK, Kourakosi P, Liakos $G$, Kremastino DT. Inverse relation of $C$ reactive protein levels to heart rate variability in patients after acute myocardial infarction. Hellenic J Cardiol 2007; 48: 64-71.
[2] Marco Ramoni, Paola Sebastiani, Richard Dybowski, Robust Outcome Prediction for Intensive-Care Patients, Methods of Information in Medicine, 40:39-45, 1999.

[3] H.R. Champion and W.J. Sacco. Measurement of patient illness severity. Critical Care Medicine, 10:552-553, 1982.

[4] Lombardi F, Sandrone G, Spinnler MT, Torzillo D, Lavezarro GC, Brusca A et al. Heart rate variability in the early hours of an acute myocardial infarction. Am Heart J 1996; 77: 1037-1044.

[5] Bigger JTJr, Fleiss JL, Steinman RC, Rolnitzky LM, Kleiger $\mathrm{RE}$, Rottman JN. Correlation among time and frequency domain measures of heart period variability two weeks after acute myocardial infarction. Am J Cardiol 1992; 69: 891-898.

[6] Vaishnav S, Stevenson R, Merchant B, Lagi K, Ranjadayalan K, Timmis AD. Relation between heart rate variability early after acute myocardial infarction and long-term mortality. Am J Cardiol 1994; 73: 653-657.

[7] Kupper NHM, Willemsen G, Van den Berg M, de Boer D, Posthuma D, Boomsma DI et al. Heritability of ambulatory heart rate variability. Circulation 2004; 110: 2792-2796.

[8] Casolo GC, Stroder P, Signorini C, Calzolari F, Zucchini M, Balli $\mathrm{E}$ et al. Heart rate variability during the acute phase of myocardial infarction. Circulation 1992; 85: 2073-2079.

[9] Singh N, Mironov D, Armstrong PW, Ross AM, Langer A. Heart rate variability assessment early after acute myocardial infarction: Pathophysiological and prognostic correlates. Circulation 1996; 93: 1388-1395.

[10] Mohammad Karimi Moridani, Majid Pouladian, Detection ischemic episodes from electrocardiogram signal using wavelet transform, J. Biomedical Science and Engineering, 2009, 2, 239-244.

[11] Alistair EW Johnson, Nic Dunkley, Louis Mayaud, Athanasios Tsanas, Andrew A Ramer, Gari D Clifford (2012). Patient Specific Predictions in the Intensive Care Unit Using a Bayesian Ensemble, Computing in Cardiology.

[12] Todd Neideen, Karen Brasel (2007). Understanding Statistical Tests, Journal of Surgical Education, Elsevier.

[13] James Robert White, Niranjan Nagarajan, Mihai Pop (2009). Statistical Methods for Detecting Differentially Abundant Features in Clinical Metagenomic Samples, PLoS Computational Biology. 